\title{
RESTRICTION OF ISOTOPIES
}

\author{
BY HERMAN GLUCK ${ }^{1}$
}

Communicated by Deane Montgomery, August 23, 1962

Let $M$ be a connected and simply connected topological manifold (with or without boundary) and $m$ a fixed point in Int $M$, the interior of $M$. Let $h_{0}$ and $h_{1}$ be two isotopic homeomorphisms of $M$, each of which leaves $m$ fixed.

It is the object of this note to show that, under these conditions, $h_{0} / M-m$ and $h_{1} / M-m$ are isotopic homeomorphisms of $M-m$.

With the standard definition of isotopy, the result follows immediately from the covering homotopy theorem, but with a somewhat more liberal (and frequently more natural) definition of isotopy, the argument is less direct. In fact in this case I can obtain the result only with the aid of the apparently irrelevant assumption that $M$ can support a piecewise linear structure.

The converse question of extending isotopies on a space to isotopies on its one-point compactification has already been answered affirmatively by R. H. Crowell [1] in the much more general setting of locally compact Hausdorff spaces.

1. Definitions. If $h_{0}$ and $h_{1}$ are homeomorphisms of $X$ onto $Y$, an isotopy between $h_{0}$ and $h_{1}$ is a continuous map

$$
H: X \times[0,1] \rightarrow Y \times[0,1]
$$

such that

(i) $H(x, 0)=\left(h_{0}(x), 0\right)$ for all $x \in X$,

(ii) $H(x, 1)=\left(h_{1}(x), 1\right)$ for all $x \in X$,

(iii) $H / X \times t$ is a homeomorphism of $X \times t$ onto $Y \times t$ for all $t \in[0,1]$.

It is shown in [1] that if $X$ is a locally compact Hausdorff space, then condition (iii) above implies

(iii') $H$ is a homeomorphism.

$H$ is called a weak isotopy between $h_{0}$ and $h_{1}$ if $H$ satisfies conditions (i), (ii) and (iii'). Thus if $X$ is locally compact and Hausdorff (in particular, if $X$ is a manifold), an isotopy is also a weak isotopy, so that isotopic homeomorphisms will also be weakly isotopic.

Weak isotopy is an important notion in the study of topological manifolds. For example, the extendability of a homeomorphism de-

1 The author holds a National Academy of Sciences Postdoctoral Research Fellowship. 
fined on the boundary of a manifold to a homeomorphism of the whole manifold depends only on the weak isotopy class of the homeomorphism.

If $M$ is a connected topological manifold and $m$ a fixed point in Int $M$, then $H(M)$ will denote the topological group of homeomorphisms of $M$ under the compact-open topology, and $H(M, m)$ the closed subgroup of homeomorphisms which leave $m$ fixed.

The projection of $M \times[0,1]$ onto $M$ will be denoted by $p r_{M}$. If $H$ is a weak isotopy between two homeomorphisms of $(M, m)$ then the curve

$$
\gamma:[0,1] \rightarrow M,
$$

defined by $\gamma(t)=p r_{M}(H(m, t))$, is a closed curve in $M$ based at $m$, which we call the trace of $H$.

\section{Restriction of isotopies.}

THEOREM 2.1. Let $M$ be a connected and simply connected topological manifold and $m$ a fixed point in Int $M$. If $h_{0}$ and $h_{1}$ are two isotopic homeomorphisms of $M$, each of which leaves $m$ fixed, then $h_{0} / M-m$ and $h_{1} / M-m$ are isotopic homeomorphisms of $M-m$.

Let

$$
H: M \times[0,1] \rightarrow M \times[0,1]
$$

be an isotopy between $h_{0}$ and $h_{1}$, and let

$$
h_{t}: M \rightarrow M
$$

be the homeomorphism of $M$ defined by

$$
H(x, t)=\left(h_{t}(x), t\right) .
$$

The following facts are well known.

(i) The map $\Gamma:[0,1] \rightarrow H(M)$, defined by $\Gamma(t)=h_{t}$, is continuous.

(ii) $H(M)$ is a principal bundle over Int $M$ with fibre and group $H(M, m)$ and projection $p: H(M) \rightarrow \operatorname{Int} M$ defined by $p(h)=h(m)$.

Then $\gamma=p \Gamma$, the trace of the isotopy $H$, is contractible because $M$ is simply connected. Hence by the covering homotopy theorem, $\Gamma$ can be deformed into a path $\Gamma^{\prime}$ which connects $h_{0}$ with $h_{1}$ and lies entirely in the fibre $p^{-1}(m)=H(M, m)$. Then

$$
H^{\prime}: M \times[0,1] \rightarrow M \times[0,1],
$$

defined by

$$
H^{\prime}(x, t)=\left(\Gamma^{\prime}(t)(x), t\right)
$$


is an isotopy between $h_{0}$ and $h_{1}$ such that, for all $t \in[0,1]$,

$$
H^{\prime}(m, t)=(m, t) \text {. }
$$

Hence $H^{\prime} /(M-m) \times[0,1]$ is an isotopy between $h_{0} / M-m$ and $h_{1} / M-m$.

3. Homma's theorem. Homma [2] has recently proved the following theorem, in the statement of which, $U_{\epsilon}\left(\widetilde{P}^{k}\right)$ denotes the set of points whose distance from $\tilde{P}^{k}$ is less than $\epsilon$.

Homma's Theorem. Let $M^{n}, \tilde{M}^{n}$ and $\tilde{P}^{k}$ be two finite combinatorial $n$-manifolds and a finite polyhedron such that $\tilde{M}^{n}$ is topologically embedded in $M^{n}, \widetilde{P}^{k}$ is piecewise linearly embedded in Int $\tilde{M}^{n}$ and $2 k+2$ $\leqq n$. Then for any $\epsilon>0$, there is an $\epsilon$-homeomorphism $F$ of $M^{n}$ onto $M^{n}$ such that

$$
F / M^{n}-U_{\epsilon}\left(\tilde{P}^{k}\right)=1,
$$

$F / \tilde{P}^{k}$ is piecewise linear.

Combining the reciprocal approximation technique employed by Homma to prove the above theorem with Lemma 2 of [2], one easily obtains the following result, which may be regarded as an indirect corollary to Homma's theorem.

THEOREM 3.1. Let $M^{n}$ be a topological $n$-manifold with boundary $B^{n-1}$. Let $M_{1}^{n}$ and $M_{2}^{n}$ be two combinatorial n-manifolds, each of which has $M^{n}$ for underlying space. Let $P_{1}$ be a polygonal arc in $M_{1}^{n}$ which meets $B_{1}^{n-1}$ only at its endpoints. If $n \geqq 4$, then for any $\epsilon>0$ there is an $\epsilon$-homeomorphism $F: M_{1}^{n} \rightarrow M_{2}^{n}$ such that

$$
\begin{aligned}
F / M_{1}^{n}-U_{\epsilon}\left(P_{1}\right) & =1, \\
F / B_{1}^{n-1} & =1,
\end{aligned}
$$

$F / P_{1}$ is piecewise linear.

\section{Restriction of weak isotopies.}

THEOREM 4.1. Let $M$ be a connected and simply connected topological manifold which can support a piecewise linear structure, and $m$ a fixed point in Int $M$. If $h_{0}$ and $h_{1}$ are two weakly isotopic homeomorphisms of $M$, each of which leaves $m$ fixed, then $h_{0} / M-m$ and $h_{1} / M-m$ are weakly isotopic homeomorphisms of $M-m$.

Since $M$ can support a piecewise linear structure, triangulate $M \times[0,1]$ as a combinatorial manifold in which $m \times[0,1]$ appears as a subcomplex. Let 


$$
H: M \times[0,1] \rightarrow M \times[0,1]
$$

be a weak isotopy between $h_{0}$ and $h_{1}$. The plan is to first find a homeomorphism $F$ of $M \times[0,1]$ onto itself such that

$$
\begin{gathered}
F /(M \times 0) \cup(M \times 1)=1, \\
F H(m \times[0,1]) \text { is polygonal, }
\end{gathered}
$$

and then a homeomorphism $F^{\prime}$ of $M \times[0,1]$ onto itself such that

$$
\begin{aligned}
F^{\prime} /(M \times 0) \cup(M \times 1) & =1, \\
F^{\prime} F H(m \times[0,1]) & =m \times[0,1] .
\end{aligned}
$$

Then $F^{\prime} F H$ will be a weak isotopy of $h_{0}$ with $h_{1}$ which takes $m \times[0,1]$ onto itself, and hence $F^{\prime} F H /(M-m) \times[0,1]$ will be a weak isotopy of $h_{0} / M-m$ with $h_{1} / M-m$.

If $\operatorname{dim} M=1, M$ is homeomorphic to an open, half-closed or closed arc, and the theorem is trivially true.

If $\operatorname{dim} M=2$, suppose first that $M$ is homeomorphic to $S^{2}$. The existence of both $F$ and $F^{\prime}$ is demonstrated in $\$ 9$ of [3]. If $M$ is not homeomorphic to $S^{2}$, then Int $M$ is homeomorphic to Euclidean 2space, $R^{2}$. The existence of $F$ is shown in $\S 9$ of [3], while the existence of $F^{\prime}$ follows from a standard argument involving Dehn's lemma [4] and the fact that an orientation preserving homeomorphism of a 2sphere is isotopic to the identity.

If $\operatorname{dim} M \geqq 3$, let $M_{2}^{n}$ be $M \times[0,1]$ triangulated as above, and let $M_{1}^{n}$ be $M \times[0,1]$ with the triangulation induced from $M_{2}^{n}$ by the homeomorphism $H$. Since $m \times[0,1]$ appears as a subcomplex of $M_{2}^{n}$, $H(m \times[0,1])$ appears as a subcomplex of $M_{1}^{n}$. Letting $P_{1}$ $=H(m \times[0,1])$, the existence of $F$ is assured by Theorem 3.1.

Since $M \times[0,1]$ is simply connected, the polygonal arc $F H(m \times[0,1])$ is homotopic to the polygonal arc $m \times[0,1]$ in $M \times[0,1]$. Since $\operatorname{dim}(M \times[0,1]) \geqq 4$, a general position argument will produce $F^{\prime}$.

5. An application. Think of $S^{n}$ as the one-point compactification of $R^{n}$ by the point $\infty$. Then the following may be regarded as a corollary to Theorem 4.1 .

THEOREM 5.1. If $h$ is a homeomorphism of $\left(S^{n}, \infty\right)$ which is weakly isotopic to the identity, then $h / R^{n}$ is weakly isotopic to the identity homeomorphism of $R^{n}$.

For the theorem is trivial when $n=1$ and $S^{n}$ is simply connected when $n>1$. 
Now let $h$ be a homeomorphism of $\left(S^{n}, \infty\right)$, and from $S^{n} \times[0,1]$ form a space $M$ by identifying $(x, 0)$ with $(h(x), 1)$ for each $x \in S^{n}$. Let $\phi: S^{n} \times[0,1] \rightarrow M$ be the decomposition map.

THEOREM 5.2. If $M$ is homeomorphic to $S^{n} \times S^{1}$, then $\phi\left(R^{n} \times[0,1]\right)$ is homeomorphic to $R^{n} \times S^{1}$.

If $M$ is homeomorphic to $S^{n} \times S^{1}$, then it follows from [5] that $h$ must be weakly isotopic to the identity. By the preceding theorem, $h / R^{n}$ must also be weakly isotopic to the identity, from which it easily follows that $\phi\left(R^{n} \times[0,1]\right)$ is homeomorphic to $R^{n} \times S^{1}$.

6. Further results. Theorem 2.1 is actually a special case of a more general result, which is briefly described below.

Let $M$ be a connected manifold and $m \in \operatorname{Int} M$. Let $h$ be a homeomorphism of $M$ leaving $m$ fixed, which is isotopic to the identity homeomorphism, $1_{M}$. Define the trace class, $\tau(h)$, to be the set of all elements of $\pi_{1}(M, m)$ which can be represented by traces of isotopies of $1_{M}$ with $h$. Then $\tau\left(1_{M}\right)$ is a central (and hence normal) subgroup of $\pi_{1}(M, m)$, and $\tau(h)$ is a coset of $\tau\left(1_{M}\right)$. Thus $\tau(h)$ may also be regarded as an element of the trace group

$$
T(M, m)=\pi_{1}(M, m) / \tau\left(1_{M}\right) .
$$

Now, if $h_{0}$ and $h_{1}$ are isotopic homeomorphisms of $M$, each of which leaves $m$ fixed, then $h_{0}^{-1} h_{1}$ is isotopic to $1_{M}$, hence $\tau\left(h_{0}^{-1} h_{1}\right)$ is defined. It then follows easily from the covering homotopy theorem applied to the bundle $H(M)$ over Int $M$ that $h_{0} / M-m$ and $h_{1} / M-m$ are isotopic homeomorphisms of $M-m$ if and only if $\tau\left(h_{0}^{-1} h_{1}\right)=\tau\left(1_{M}\right)$.

This condition is automatically satisfied when $M$ is simply connected, hence Theorem 2.1.

Theorem 4.1 follows from a similar result about weak isotopy.

\section{REFERENCES}

1. R. H. Crowell, Invertible isotopies, Proc. Amer. Math. Soc. (to appear).

2. T. Homma, On the imbedding of polyhedra in manifolds, (to appear).

3. H. Gluck, The embedding of two-spheres in the four-sphere, Trans. Amer. Math. Soc. 104 (1962), 308-333.

4. C. D. Papakyriakopoulos, On Dehn's lemma and the asphericity of knots, Ann. of Math. 66 (1957), 1-26.

5. M. Brown and H. Gluck, Stable structures on manifolds. I. Homeomorphisms of $S^{n}$, (to appear).

The Institute for Advanced Study 